\title{
Necrotizing Funisitis
}

National Cancer Institute

\section{Source}

National Cancer Institute. Necrotizing Funisitis. NCI Thesaurus. Code C117323.

A ring of karyorrhectic debris that may exhibit dystrophic mineralization and/or

identifiable fetal neutrophil infiltrate in Wharton's jelly that is oriented towards the amniotic surface. The cord has a denser ring externally and a fainter ring centrally. 